\title{
CAPÍTULO 18: APLICAÇÃO DE LACASES EM SÍNTESE ORGÂNICA PARA OBTENÇÃO DE BIOATIVOS COM ATIVIDADE ANTIOXIDANTE: UMA REVISÃO
}

\section{CHAPTER 18: APPLICATION OF LACCASES IN ORGANIC SYNTHESIS AND OBTAINMENT OF BIOACTIVES WITH ANTIOXIDANT ACTIVITY: A REVIEW}

\author{
Camila Gabriel Kato-Schwartz ; Emanueli Backes²; Rosane Marina Peralta ${ }^{3}$; Gisella Maria Zanin ${ }^{4}$
}

\begin{abstract}
Resumo
As lacases (EC 1.10.3.2) são enzimas capazes de catalisar a oxidação de diferentes fenóis produzindo radicais fenoxi concomitantemente reduzindo o oxigênio molecular à água. Devido aos benefícios dos processos oxidativos com lacase, estas enzimas têm sido utilizadas nas indústrias de alimentos, têxtil, papel e celulose e são utilizadas também em biossensores e em processos de biorremediação. O aumento da demanda por compostos antioxidantes altamente ativos e estáveis para aplicação na indústria de alimentos tem impulsionado a investigação de novos métodos para síntese de antioxidantes. Dessa forma, o objetivo deste trabalho foi revisar aspectos relacionados à aplicação das lacases em reações de síntese orgânica via oligomerização/polimerização de diferentes fenólicos visando a obtenção de novos bioativos antioxidantes. As características e fontes das lacases utilizadas bem como a estrutura e propriedades dos novos compostos fenólicos sintetizados foram discutidos em detalhes. Foi dada uma atenção especial aos métodos de avaliação da atividade antioxidante e os benefícios da utilização das enzimas imobilizadas nos bioprocessos.
\end{abstract}

Palavras-Chave: Lacase, Basidiomiceto, Síntese orgânica, Antioxidantes.

\begin{abstract}
Laccases (EC 1.10.3.2) are enzymes capable of catalyzing the oxidation of various phenols by producing phenoxy radicals with concomitant reduction of molecular oxygen to water. Due to the benefits of oxidative processes with laccase, these enzymes have been used in the food, textile, paper and cellulose industries and are also used in biosensors and in bioremediation processes. The increase in the necessity for antioxidant compounds with improved properties for application in the food industry has driven the investigation of promising methods such as the application of laccase for the synthesis of antioxidants. Within this context, the objective of the present work is to review aspects related to the application of laccases in reactions of organic synthesis via oligomerization/polymerization of phenolics, aiming at obtaining new antioxidant bioactives. The characteristics and sources of the laccases as well as the structure and properties of the phenolic compounds are discussed in detail. Special attention was paid to methods of assessing antioxidant activity and the benefits of using immobilized enzymes in bioprocesses.
\end{abstract}

Keywords: Laccase, Basidiomycetes, Organic synthesis, Antioxidants.

\footnotetext{
${ }^{1}$ Programa de Pós-graduação em Engenharia Química, Universidade Estadual de Maringá, camilagkato@hotmail.com

${ }^{2}$ Programa de Pós-graduação em Ciência de Alimentos, Universidade Estadual de Maringá, emanuelibackes@outlook.com

${ }^{3}$ Programa de Pós-graduação em Ciência de Alimentos, Universidade Estadual de Maringá, rmperalta@uem.br

${ }^{4}$ Programa de Pós-graduação em Engenharia Química, Universidade Estadual de Maringá, giselladeq@ gmail.com
} 


\section{Introdução}

Compostos fenólicos ou polifenólicos vegetais são um amplo grupo de metabólitos que se originam do metabolismo secundário das plantas. Eles contêm um ou mais grupos hidroxila ligados a um anel de benzeno e têm um papel importante na defesa contra patógenos de plantas e agentes estressores abióticos (DAGLIA, 2012). Os compostos fenólicos estão se tornando cada vez mais objeto de intensa pesquisa devido às suas propriedades bioativas, incluindo as atividades antioxidante, antidiabética, anti-inflamatória, antialérgica e antiplaquetária. Os compostos fenólicos atuam na prevenção da oxidação lipídica em alimentos e na prevenção de doenças como câncer e aterosclerose que são induzidas por radicais livres (XIAO et al., 2016; XIAO, 2017; SINGH, et al., 2018).

Os compostos fenólicos naturais com atividade antioxidante estão presentes em baixas concentrações em relação a um substrato oxidável e recebem atenção pela capacidade de retardar ou inibir a oxidação desse substrato (ADELAKUN et al., 2012b). Muitos dos compostos fenólicos naturais são sensíveis à temperatura, $\mathrm{pH}$ e à luz devido à presença de duplas ligações alternadas (NEMADZIVA et. al., 2018). Consequentemente, os mais diversos tipos de vegetais, comestíveis e não comestíveis tem sido utilizado como matrizes na pesquisa de novos compostos fenólicos mais ativos e mais estáveis. Além desta busca pela descoberta de novos fenólicos naturais, uma segunda abordagem tem sido empregada na obtenção destes fenólicos mais ativos e mais estáveis: modificações estruturais em fenólicos já conhecidos.

A síntese orgânica utilizando um composto fenólico ou uma mistura de compostos fenólicos com o objetivo de obtenção de novos compostos com propriedades funcionais mais elevadas que os fenólicos parentais, ganha um componente ambiental importante quando realizada utilizando-se como catalisador do processo uma enzima. O apelo ambiental é o grande impulsionador desta abordagem, pois possibilita a modificação dos substratos sem o uso de substâncias químicas e em condições brandas, com baixo impacto ambiental. Os radicais formados pela ação dos biocatalisadores resultam, geralmente, na formação de novas moléculas com propriedades biológicas de interesse (ADELAKUN et al., 2012b; MUNIZ-MOURO et al., 2017).

As lacases (EC 1.10.3.2) são cobre-oxidases que oxidam substratos fenólicos formando radicais fenoxil. As lacases são encontradas em fungos, bactérias, plantas e insetos (NARAYANAN et al., 2015; DEVASIA e NAIR 2016; REZAEI et al., 2017). Lacases livres e imobilizadas vem sendo aplicadas em diversas áreas incluindo degradação de poluentes, cosmética, bioenergética e síntese orgânica (NEMADZIVA et al., 2018; MUÑIZ-MOURO et al. 2018; REANO et al., 2016; NAGHDI et al., 2019; DARONCH et al., 2020). 
O objetivo deste trabalho foi revisar aspectos relacionados à aplicação das lacases em reações de síntese orgânica via oligomerização/polimerização de diferentes fenólicos visando a obtenção de novos bioativos antioxidantes. As características e fontes das lacases utilizadas bem como a estrutura e propriedades dos novos compostos fenólicos sintetizados foram discutidos em detalhes. Foi dada uma atenção especial aos métodos de avaliação da atividade antioxidante e os benefícios da utilização das enzimas imobilizadas nos bioprocessos.

\section{Metodologia empregada}

Para a seleção dos artigos científicos, utilizou-se as seguintes bases de dados: Scopus e Web of Science considerando os artigos publicados nos últimos 10 anos (2011-2020) e utilizando como termos de buscas os termos "lacase" e "síntese" e "antioxidante" (em inglês laccase, synthesis e antioxidant, respectivamente). Foram encontrados 7524 artigos na busca com a palavra laccase e 52 artigos quando a busca foi realizada com as os três termos (laccase synthesis e antioxidant) na base Scopus e 7821 artigos (laccase) e 59 artigos (laccase, synthesis e antioxidant). Foram considerados para esta revisão, somente os artigos que possibilitaram aos autores alcançar os objetivos pré-estabelecidos.

\section{Desenvolvimento}

\subsection{Lacase: características e fontes}

As lacases são enzimas amplamente distribuídas entre os seres vivos, sendo encontradas em plantas, vegetais e insetos. São encontradas em múltiplas formas e isso explica as diversas funções desta enzima. Nas plantas, as lacases atuam na polimerização da lignina e na síntese de poliflavonóides. Já nos insetos, a lacase tem como função principal a esclerotização da cutícula vegetal. Quando proveniente de bactérias, lacases são relacionadas aos processos de síntese de pigmentos marrons nos esporos e proteção contra a luz UV. Também possuem importantes funções na homeostase do íon cobre e na morfogênese (CHAUHAN; GORADIA; SAXENA, 2017; SHARMA et al., 2018; JANUSZ et al., 2020).

O primeiro relato da ocorrência de lacase foi feito em 1883 através de um estudo da secreção leitosa, urushi, árvore chinesa/japonesa Rhus vernicifera (GAVNHOLT; LARSEN, 2002; JANUSZ et al., 2020). Lacases tem sido isoladas de plantas como feijão, pinho, mung e ameixa e também da família botânica de Anacardiaceae, contudo, ainda é escasso o uso de lacases vegetais em aplicações industriais (WANG et al., 2015; JANUSZ et al., 2020). Lacases são também encontradas em vários gêneros de insetos, como Calliphora, Diploptera, Bombyx, Drosophila, Lucilia, Menduca, Musca, Phormia, Rhodnius, Oryctes, Papilio e Sarcophaga 
(CHAUHAN; GORADIA; SAXENA, 2017; JANUSZ et al., 2020), mas assim como as lacases vegetais, não são, até o presente momento utilizadas em bioprocessos.

A primeira lacase isolada a partir de uma bactéria (Azospirillum lipoferum) ocorreu somente em 1993 a partir da rizosfera de arroz e desde então, elas foram identificadas em outras bactérias Gram-positivas como Staphylococcus, Streptomyces, Geobacillus, Rhodococcus, Bacillus, Azospirillum, Aquisalibacillus e Lysinibacillus (DHIMAN; SHIRKOT 2015; NARAYANAN et al. 2015; REZAEI et al., 2017). Algumas bactérias Gram-negativas também apresentam a capacidade de secretar a lacase, como é o caso das Pseudomonas, Enterobacter, Proteobacterium, Delfia and Alteromonas (DHIMAN; SHIRKOT 2015; DEVASIA; NAIR 2016; CHAUHAN; GORADIA; SAXENA, 2017). As lacases de bactérias são consideradas mais estáveis a elevadas temperaturas e $\mathrm{pH}$ quando comparadas as fúngicas e possuem um peso molecular que varia de 20 a 80 kDa (CHAUHAN; GORADIA; SAXENA, 2017).

As lacases mais estudadas são as encontradas nos fungos basidiomicetos causadores da decomposição branca da madeira, tais como Pleurotus ostreatus, Trametes versicolor, Oudemansiella canarii, Pycnoporus cinnabarinus, Pycnoporus sanguineus etc., além de diversos ascomicetos e alguns deuteromicetos, como Trichoderma sp. (RIVERA-HOYOS et al., 2013; CHAUHAN; GORADIA; SAXENA, 2017; BRUGNARI et al., 2018; JANUSZ et al., 2020).

As lacases fúngicas desempenham papel importante na pigmentação e resistência dos esporos e estão associadas à renovação do húmus, aos processos de desintoxicação, formação de corpos e principalmente biodegradação da lignina (ZHANG et al., 2015; CHAUHAN; GORADIA; SAXENA, 2017; SHARMA et al., 2018; JANUSZ et al., 2020). Percebe-se que a função da lacase é sintética em quase todos os organismos, com exceção dos fungos, onde além da função de síntese de pigmentos, por exemplo, também estão associadas à processos de degradação, como a degradação da lignina. Ela também foi a primeira enzima que se mostrou capaz de polimerizar monômeros de lignina in vitro (GAVNHOLT; LARSEN, 2002).

Como já abordado, as lacases são capazes de catalisar reações de oxidação monoeletrônica de diversos substratos tais como anilinas, fenóis, aminas aromáticas, aminofenóis, metoxifenóis, polifenóis, poliaminas e derivados de lignina. Concomitantemente ocorre a produção de água sendo o único subproduto da oxidação monoeletrônica de substratos que utiliza o oxigênio molecular (Figura 1) (JEON et al., 2010; BRUGNARI et al., 2018; WELLINGTON; GOVINDJEE; STEENKAMP, 2018). 
Figura 1. Oxidação direta da lacase na transformação do substrato fenólico catecol em $o$-benzoquinino.

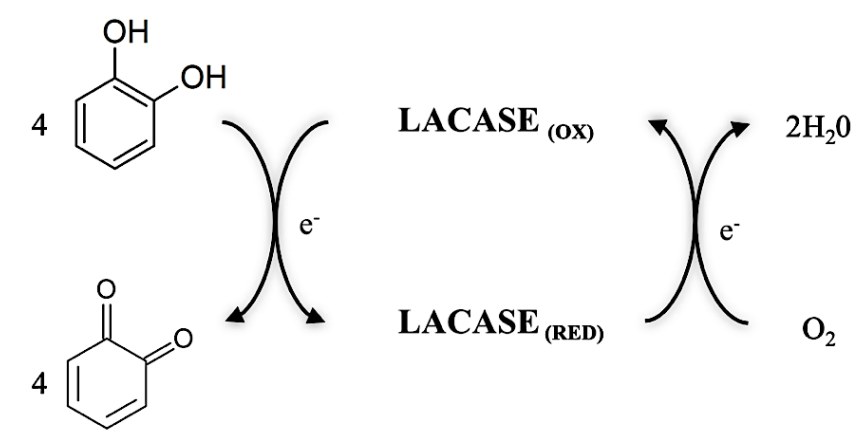

Fonte: Modificado de Polak e Jarosz-Wilkolazca (2012).

Devido a sua atividade oxidativa de largo espectro, as lacases são amplamente utilizadas em diversas aplicações industriais, incluindo modificação de polímeros, branqueamento têxtil, processamento de alimentos, síntese orgânica, indústria cosmética e transformação e biorremediação de uma infinidade de compostos xenobióticos incluindo hidrocarbonetos aromáticos policíclicos, pesticidas, corantes sintéticos e produtos farmacêuticos como diclofenaco (Figura 2). Devido a extensa aplicação e utilização dessas enzimas, elas podem ser consideradas um dos biocatalisadores mais importantes e com aplicações industriais divergentes como o potencial de polimerizar e despolimerizar (BILAL et al., 2019; TAHERAN et al., 2017; BARRIOS-ESTRADA et al., 2018). Este aspecto foi comprovado pelo elevado número de artigos encontrados na pesquisa que abordou o período de 2011-2020 realizada nas bases Scopus (7524 artigos) e Web of Science (7821) utilizado nesta revisão.

Figura 2. Principais áreas de aplicação de lacases.

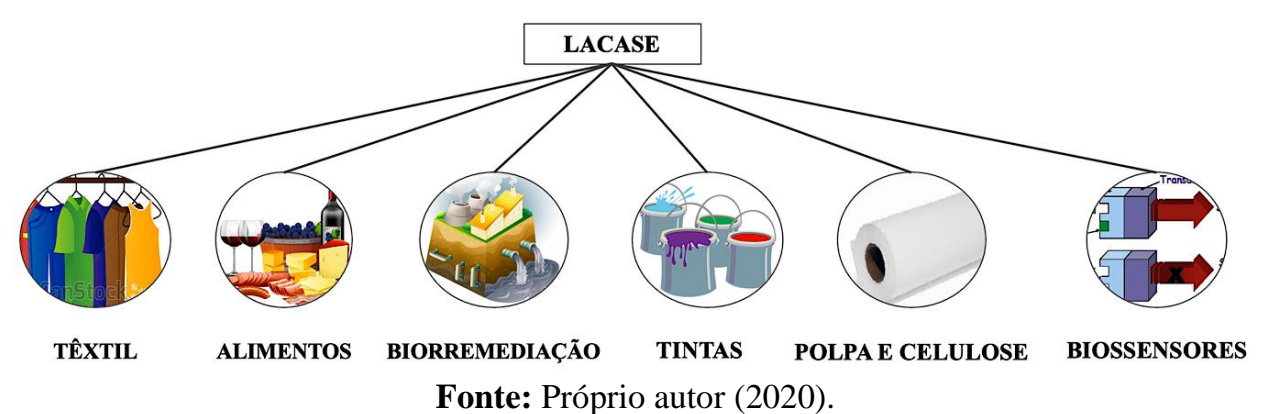

\subsection{Compostos fenólicos: estrutura e propriedades}

Os compostos fenólicos, muitos deles metabólitos secundários, constituem uma classe extremamente importante de moléculas. Esses compostos ocorrem naturalmente em frutas e vegetais e estão relacionados à efeitos benéficos à saúde com propriedades antidiabética, antiinflamatória, antialérgicos, antiplaquetários e antitumorais (XIAO et al., 2016; XIAO, 2017; 
SINGH, et al., 2018). Também exercem função como eliminadores de radicais livres, inibidores da oxidação de lipoproteínas e agente quelantes de metais (MAMEDE; PASTORE, 2004; PREHN; GONZALO-RUIZ; CORTINA-PUIG, 2012).

A presença de compostos fenólicos em alimentos influencia a qualidade dos vegetais como aroma, sabor, cor e atributos sensoriais como vinhos, café, sucos e chás (AGRAWAL; CHATURVEDI; VERMA, 2018; LETTERA et al., 2016; PEZZELLA et al., 2015). Estes compostos são amplamente utilizados como aditivos em uma variedade de processos industriais, como na indústria de papel, na fabricação de plásticos, corantes ou drogas (PREHN; GONZALO-RUIZ; CORTINA-PUIG, 2012).

Com base em elementos estruturais que ligam anéis benzeno e números de grupos hidroxila ligados, podem ser classificados em fenóis simples ou polifenóis (SINGH, et al. 2018). Por sua vez, os polifenóis (fitoquímicos poli-hidroxilados) que possuem estruturas comuns podem ser divididos em duas categorias, flavonóides (polifenóis) e polifenóis não flavonóides (fenóis simples ou ácidos) (PREHN; GONZALO-RUIZ; CORTINA-PUIG, 2012; SINGH, et al., 2018).

A estrutura química dos flavonóides favorece sua ação antioxidante pois possuem dois ou mais anéis aromáticos, cada um contendo um ou mais grupos hidroxila fenólicos e são conectados através de uma ponte de carbono fornecendo assim, compostos com alta atividade antioxidante. Esses compostos são sensíveis à oxidação enzimática e não enzimática e a atividade antioxidante é sensível à luz devido as suas duplas ligações alternadas (MAMEDE; PASTORE, 2004; HELENO et al., 2015; XIAO et al., 2016; XIAO, 2017; SINGH, et al., 2018).

Os polifenóis não flavonóides conhecidos como ácidos fenólicos podem ser divididos em dois grupos principais, ácidos hidroxibenzóicos que são derivados do ácido benzóico e ácidos hidroxicinâmicos, derivados de ácidos cinâmico. Quimicamente, esses compostos possuem ao menos um anel aromático e pelo menos um hidrogênio é substituído por um grupo hidroxila e ambos os grupos possuem propriedades antioxidantes (HELENO et al., 2015).

Os derivados de ácidos hidroxicinâmicos possuem um anel aromático com uma cadeia carbônica, formado por 3 carbonos ligados ao anel. Dentro desse grupo estão presentes os ácidos p-cumárico,caféico, ferúlico e sinápticos sendo encontrados na natureza (DEGÁSPARI; WASZCZYNSKYJ, 2004).

Os ácidos hidroxibenzóicos possuem grupo carboxílico ligado ao anel aromático e, dentro desse grupo, estão presentes os ácidos gálico, elágico, vanílico, siríngico, entre outros. A atividade antioxidante desses compostos está relacionada aos seus ésteres, contudo, também 
é determinada pelo número de hidroxilas presente na molécula e a posição (DEGÁSPARI; WASZCZYNSKYJ, 2004; MAMEDE; PASTORE, 2004).

Os ácidos sináptico, ferúlico e p-cumárico são antioxidantes mais ativos do que os derivados do ácido benzóico, tais como ácido procatecuíco, siríngico e vanílico. Isso se deve à dupla ligação presente na molécula dos derivados do ácido cinâmico $(-\mathrm{HC}=\mathrm{CHCOOH})$, que participa da estabilização do radical por ressonância de deslocamento do elétron desemparelhado, enquanto que os derivados do ácido benzóico não apresentam essa característica (DEGÁSPARI; WASZCZYNSKYJ, 2004).

O ácido ferúlico contém uma hidroxila na posição orto com o grupo metoxila sendo doador de elétrons, um fator responsável por aumentar a estabilidade do radical fenoxil e também do aumento da eficiência antioxidante do ácido ferúlico. Contudo, ácidos que contenham uma segunda hidroxila na posição orto ou para tem-se aumento da atividade antioxidante, como acontece com o ácido caféico. Isso explica a maior atividade antioxidante presente no ácido caféico quando comparado ao ácido ferúlico (DEGÁSPARI; WASZCZYNSKYJ, 2004). Entretanto, a atividade antioxidante do ácido caféico é próxima a do ácido m-cinâmico, mesmo possuindo um grupo de hidroxila a mais (MAMEDE; PASTORE, 2004).

O ácido $p$-cumárico possui o grupo hidroxila na posição para o que lhe proporciona maior atividade antioxidante em relação ao ácido hidrocinâmico (MAMEDE; PASTORE, 2004). Os ácidos ferúlico, p-cumárico e sináptico possuem elevada propriedade antioxidante quando comparados aos derivados do ácido benzóico como os ácidos vanílico e siríngico devido à dupla ligação presente na molécula dos derivados do ácido cinâmico $(-\mathrm{HC}=\mathrm{CHCOOH}) . \mathrm{O}$ ácido cinâmico está relacionado a estabilização do radical devido a ressonância de deslocamento do elétron desemparelhado. Já os derivados do ácido benzóico não possuem essa característica (DEGÁSPARI; WASZCZYNSKYJ, 2004).

Existem compostos fenólicos que não se apresentam na forma livre no tecido vegetais, como é o caso dos taninos que estão presentes na forma de polímeros. Os taninos possuem alto peso molecular e podem ser classificados em relação ao seu tipo estrutural em: taninos hidrolisáveis e condensados. Os taninos hidrolisáveis possuem um núcleo central de álcool poliédrico ou glicose, esterificado com ácido elágico ou gálico, e são hidrolisáveis com ácidos, bases ou enzimas. Já os taninos condensados são polímeros de catequina ou leucoantocianidina e não são hidrolisáveis por com ácidos (SOARES, 2002; KATO et al., 2017).

Os benefícios biológicos proporcionados pelos compostos fenólicos estão diretamente relacionados com a atividade antioxidante que cada fenol exerce sobre determinado meio. A 
ação antioxidante do composto fenólico depende de sua estrutura química, podendo ser estipulado pela ação da molécula como agente redutor (potencial de quelação de metais, reatividade com outros antioxidantes e velocidade de inativação do radical livre) (XIAO et al., 2016; XIAO, 2017; SINGH, et al., 2018).

\subsection{Método de avaliação da atividade antioxidante}

A capacidade antioxidante pode ser determinadas por diversos métodos e expressa por meio de diversos parâmetro como através da capacidade de redução de metal (FRAP - ferric reducing antioxidant power, CUPRAC - cupric ion reducing antioxidant capacity), remoção de um radical peroxil (TRAP - total reactive antioxidant potential, ORAC - oxygen radical absorbance capacity), atividade sequestrante do DPPH (2,2-difenil-1-picrylhydrazil), atividade sequestrante do ABTS (2,20-azino-bis (ácido 3-ethylbenzthiazoline-6-sulfônico) e através da quantificação de produtos formados durante a peroxidação de lipídeos lipídeos (TBARS, oxidação do LDL (lipoproteínas de baixa densidade) e co-oxidação do $\beta$-caroteno) (SUCUPIRA et al., 2012; ALVES et al., 2010). Para a determinação da capacidade antioxidante in vivo, os métodos mais utilizados são o DPPH, ORAC, ABTS e FRAP.

A atividade antioxidante de compostos fenólicos como os flavonóides pode ser determinada através da reatividade como agente doador de $\mathrm{H}$ e elétrons, reatividade diante a outros antioxidantes, estabilidade do radical flavanoil formado, quanto a capacidade de quelar metais de transição e através da solubilidade e interação com membranas.

O método do radical DPPH se baseia no sequestro de radicais livres, no caso o DPPH, no qual possui um elétron desemparelhado por toda a molécula conferindo uma coloração violeta. Dessa forma, esse método se baseia na capacidade antioxidante de uma determinada molécula em sequestrar o radical livre DPPH. Quando isso ocorre, tem-se a mudança na coloração de violeta para amarelo pálido (SUCUPIRA et al., 2012; ALVES et al., 2010; ZIYATDINOVA; SNEGUREVA; BUDNIKOV, 2017).

O método ORAC se baseia na propriedade fluorescente das proteínas B-ficoeritrina (B$\mathrm{PE})$ e R-ficoeritrina (R-PE) no qual tem-se um decréscimo da fluorescência dessas proteínas, devido a perda de sua conformidade ao sofrer danos oxidativo (SUCUPIRA et al., 2012; ALVES et al., 2010).

$\mathrm{O}$ método do ABTS está relacionado à habilidade dos antioxidantes em sequestrar o cátion $\mathrm{ABTS}^{\bullet+}$. Esta captura resulta em um decréscimo na absorbância, sendo lida a partir da mistura do radical com o antioxidante em diferentes tempos (SUCUPIRA et al., 2012). 
No método FRAP, complexo férrico-tripiridiltriazina (FeIII-TPZ) é reduzido ao complexo ferroso (FeII-TPZ) quando as condições do meio reacional são ácidas e na presença de um antioxidante. O complexo formado devido a esta reação indica uma coloração azul intensa e com absorção máxima de 593 nm (BENZIE; STRAIN, 1996).

Como há uma gama enorme de tipos de radicais livres, não há ainda um único método capaz de avaliar a atividade antioxidante de forma precisa e quantitativa. Por conta disso, vários métodos devem ser utilizados na avaliação dos diversos fenólicos sejam eles naturais ou obtidos através de síntese orgânica.

\subsection{Modificação de compostos fenólicos por reações de síntese orgânica e avaliação da atividade antioxidante}

Embora o uso de compostos fenólicos tenha sido impulsionado pelo apelo nutracêutico de suas multifacetadas atividades biológicas, fatores como baixa solubilidade em meio aquoso e suscetibilidade ao calor, luz e oxigênio podem limitar suas aplicações (NEMADZIVA et al., 2018). Ademais, a atividade antioxidante destes compostos é, em geral, inferior à de compostos antioxidantes sintéticos (ADELAKUN et al., 2012a).

Compostos fenólicos monoméricos simples podem apresentar meia-vida curta no organismo, além de serem metabolizados em estruturas menos ativas (NEMADZIVA et al., 2018). Consequentemente, cresce o interesse em abordagens biocatalíticas, como a síntese "verde", que permitam a oligomerização de polifenóis e formação de oligômeros com propriedades bioativas intensificadas. Reações de polimerização enzimática são capazes de resultar em polifenóis com densidade eletrónica superior à unidade monomérica, maior solubilidade, termoestabilidade e propriedades antioxidantes (MUNIZ-MOURO et al., 2017; MUÑIZ-MOURO et al., 2018). Diversos flavonoides de alto peso molecular exibem propriedades fisiológicas aprimoradas, como atividade antioxidante e anticarcinogênica, e um tempo de circulação relativamente mais longo in vivo (KURISAWA et al., 2003).

$\mathrm{O}$ interesse especial em lacases como catalisador dos processos de biotransformação provém da enzima utilizar oxigênio molecular como aceptor final de elétrons, diferentemente de peroxidases, por exemplo, que requerem peróxido de hidrogênio (MUÑIZ-MOURO et al. 2018). Para além, lacase são especialmente capazes de oxidar diversos compostos aromáticos, produzindo radicais intermediários altamente reativos que se ligam através de reações de dimerização e trimerização, por exemplo (MOGHARABI; FARAMARZI, 2014). O interesse na síntese orgânica de dímeros e trímeros de compostos fenólicos através de reações 
enzimáticas cresce dado o valor terapêutico associado a estes produtos de acoplamento (ADELAKUN et al., 2012a).

Em estudo conduzido por Adelakun et al. (2012a), dois produtos diméricos $(\beta-5$ e $\beta$ - $\beta$ ) foram obtidos da reação de modificação do ácido ferúlico catalisada por lacase de Trametes pubescens. O dímero $\beta-5$ apresentou atividade antioxidante amplificada comparativamente ao substrato ácido ferúlico, medida pelos métodos DPPH e ABTS. Este comportamento está relacionado, muito provavelmente, ao aumento de grupos doadores de elétrons e à formação do grupo ácido carboxílico com uma ligação dupla $\mathrm{C}-\mathrm{C}$ insaturada adjacente, que fornece locais de ataque adicionais para os radicais (Figura 3).

Figura 3. Via da reação proposta para a síntese do dímero $\beta-5$ de ácido ferúlico catalisada por lacase.

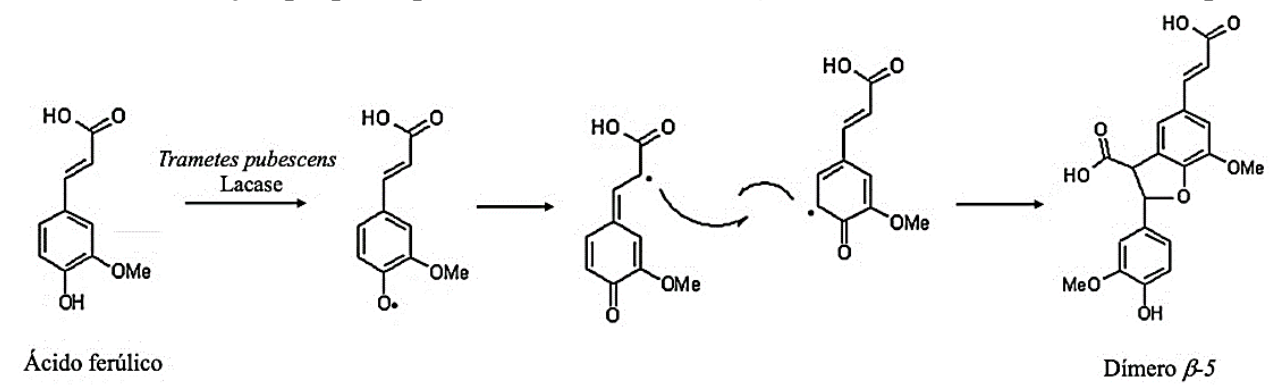

Fonte: Modificado de Adelakun et al. (2012a).

A oxidação do grupamento $p$-hidroxila do composto fenólico pela ação da lacase produz um radical que marca o início da reação. A estabilização por ressonância pode levar o elétron não emparelhado à ocupar diferentes posições na molécula radicalizada. Se o acoplamento deste elétron na posição $\beta$ se der com outro na posição C5 do próximo radical, haverá a formação de um dímero através da ligação $\beta$-5. Da mesma forma o acoplamento covalente entre elétrons desemparelhados ambos na posição $\beta$ resulta na formação de um dímero $\beta$ - $\beta$. Este comportamento é corriqueiramente observado em reações de dimerização, embora na natureza sejam as ligações $\beta-O-4$ as predominantemente formadas na síntese de polímeros (ADELAKUN et al., 2012a; NEMADZIVA et al., 2018).

De forma análoga, o dímero $\beta$ - $\beta$ do ácido caféico foi sintetizado utilizando uma lacase de Streptomyces coelicolor A3(2) (Figura 4). O produto de síntese demonstrou aumento de 1,5 vezes na capacidade de sequestro do radical livre DPPH e atividade antioxidante equivalente ao Trolox aumentada em 1,8 vezes, comparativamente ao ácido cafeico. As propriedades de solubilidade aquosa e estabilidade à pH ácido foram melhoradas (NEMADZIVA et al., 2018). 
Figura 4. Via de reação proposta para a síntese de phellinsin A catalisada por lacase.

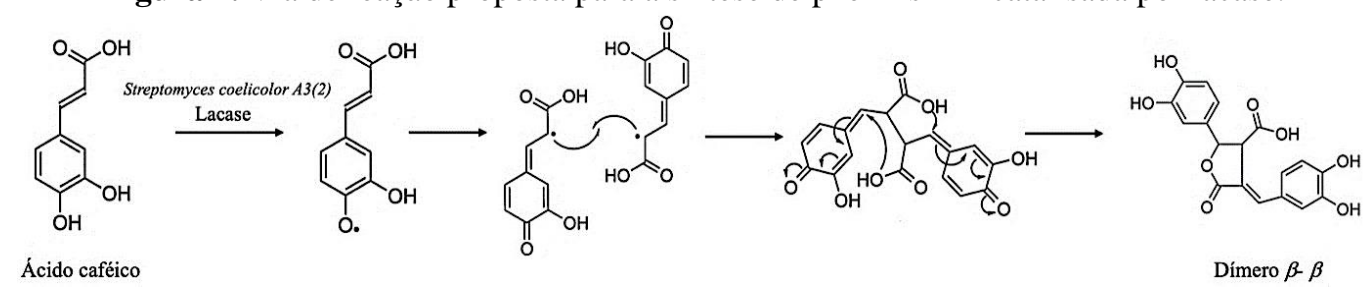

Fonte: Modificado de Nemadziva et al. (2018).

Dado o grande interesse nas propriedades dos compostos de síntese, condições operacionais otimizadas são fundamentais para configurar um processo eficiente e econômico. A polimerização do monômero de rutina foi previamente elucidada por Kurisawa et al. (2003). O polímero demonstrou atividade de eliminação de superóxido e inibição na oxidação de lipoproteína de baixa densidade humana (LDL) ampliadas. Entretanto, estudos adicionais conduzidos por Muñiz-Mouro et al. (2018) demonstraram que o oligômero de rutina provenientes de reação com lacase com baixa atividade enzimática inicial obteve melhores características nutracêuticas. $\mathrm{O}$ aumento da atividade enzimática também influenciou negativamente a estabilidade térmica do produto. Doses mais elevadas de enzimas promoveram a formação de múltiplas ligações intermoleculares entre as unidades de rutina, o que reduziu o conteúdo de fenólicos na molécula, com consequente queda na atividade antioxidante (HEIM; TAGLIAFERRO; BOBILYA, 2002).

A oxidação do composto fenólico 2,6 dimetoxifenol por lacase de Trametes pubescens gerou, como produto principal, um dímero simétrico do substrato obtida de uma ligação $\mathrm{C}-\mathrm{C}$ (3,3',5,5'-tetrametoxi-bifenil-4,4'-diol) (ADELAKUN et al. 2012b). De acordo com os ensaios DPPH e FRAP, o dímero demonstrou atividade antioxidante aproximadamente duas vezes maior do que o substrato fenólico. Os resultados sustentam a potencialidade da aplicação da lacase na síntese de compostos nutracêuticos com interesse comercial.

Reano et al. (2016) demonstraram o uso de lacase comercial oriunda de Trametes versicolor na síntese de compostos para utilização como polímeros de embalagens de alimentos. A reação procedeu com a oligomerização de bisfenóis à base de ácido diidroferúlico renováveis para síntese de novos oligômeros com elevadas atividades antioxidantes. Esses oligômeros fenólicos alifáticos - aromáticos, com porções do tipo bisguaiacol, exibiram alta estabilidade térmica, bem como potente atividade antioxidante demonstrada pelo método DPPH.

Apesar dos diversos estudos evidenciando a ação eficiente da lacase em processos de síntese de compostos com valor agregado, estes são conduzidos em escala laboratorial. A falta de estabilidade operacional por períodos prolongados e a dificuldade de recuperação e reuso 
das enzimas quando na forma livre, fazem sua aplicação em escala industrial ser limitada. Dessa forma, a imobilização enzimática vem sendo empregada como alternativa eficaz para transpor tais empecilhos e produzir biocatalisadores industriais robustos (VELASCO-LOZANO et al., 2015).

\subsection{Imobilização de lacase: alternativa para melhorar o desempenho reacional das lacases}

A imobilização enzimática consiste na restrição da mobilidade celular através de interações químicas ou físicas à uma fase (matriz ou suporte) diferente daquele para substratos e produtos (BOUABIDI; EL-NAAS; ZHANG, 2019). Enquanto as interações físicas se baseiam na adsorção ou aprisionamento da porção enzimática, as interações químicas se estabelecem por ligações covalentes à um suporte ou ligações cruzadas (reticulação) (Figura 5) (DARONCH et al., 2020).

Quando imobilizada, a lacase apresenta aumento da estabilidade, com proteção da estrutura da enzima à condições adversas, maior durabilidade e possibilidade de reuso acrescido. Tais fatores se traduzem em redução dos custos operacionais e aumento na eficiência do sistema biocatalítico (VELASCO-LOZANO et al., 2015; NAGHDI et al., 2019; DARONCH et al., 2020).

Figura 5. Esquema ilustrativo da imobilização enzimática por adsorção em suporte (a), encapsulamento (b), ligação covalente ao suporte (c) e ligação cruzada (d).

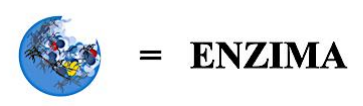

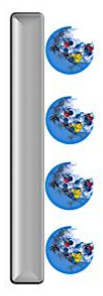

(a)

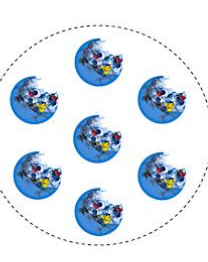

(b)

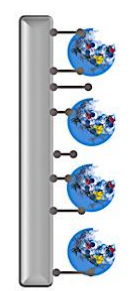

(c)

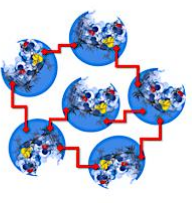

(d)

Fonte: Próprio autor (2020).

Vários métodos já foram reportados para imobilização eficaz de lacase, nomeadamente imobilização em polímeros, imobilização com quitosana (NAGHDI et al., 2019) e suporte de agarose anionicamente ativado (BRUGNARI et al., 2018). Portadores multifários também têm sido estudados para a imobilização da lacase (ZHOU et al., 2013; LIU et al., 2012), entre outros.

Lacases imobilizadas já provvaram ser uma poderosa ferramenta para produção de biossensores industriais (CABALLERO et al., 2018; MOHTAR et al., 2019), degradação de 
poluentes ambientais (RATANAPONGLEKA; PUNBUT, 2017; VIDAL-LIMON et al., 2018), deslignificação de biomassa lignocelulósica (AMIN et al., 2018; ASGHER et al., 2018) e melhoramento de características sensoriais e tecnológicas de alimentos e bebidas (HOU et al., 2016; LETTERA et al., 2016). Entretanto, enquanto a pesquisa e aplicação de lacase livre na síntese de novos compostos orgânicos com propriedades bioativas é já realidade, há uma escassez bibliográfica em estudos que elucidem seu comportamento quando na forma imobilizada. Os resultados promissores da aplicação deste biocatalisador nos mais distintos segmentos industriais, aliado aos avanços de técnicas de imobilização enzimática, demanda que esta lacuna científica seja melhor explorada e elucidada.

\section{Considerações Finais}

A versatilidade bioquímica da lacase se deve à sua capacidade de oxidação de uma ampla gama de substratos. O interesse nestas enzimas como biocatalisadores em reações de oligomerização e polimerização de monômeros fenólicos é crescente devido à possibilidade de melhoria das atividades biológicas dos compostos formados em relação aos compostos de origem. Os produtos reacionais sintetizados são úteis para aplicações em indústrias de alimentos, farmacêutica e cosmética dada a notável atividade antioxidante dos mesmos. Ademais, esta é uma abordagem ambientalmente correta que se enquadra no conceito de "química verde".

\section{Agradecimentos}

Os autores Camila Gabriel Kato Schwartz (Bolsa PDJ-Proc. 151189/2019-6), Emanueli Backes (bolsa-doutorado) e Rosane Marina Peralta (Proc. 304406/2019-8 e Proc. 404898/20165) agradecem o apoio financeiro do Conselho Nacional de Desenvolvimento Científico e Tecnológico (CNPq).

\section{Referências}

ADELAKUN, O.E.; KUDANGA, T.; PARKER, A.; GREEN, I.R.; ROES-HILL, M.L.; BURTON, S.G. Laccase catalyzed dimerization of ferulic acid amplifies antioxidant activity, Journal of Molecular Catalysis B: Enzymatic, v. 74, p. 29-35, 2012a.

ADELAKUN, O.E.; KUDANGA, T.; GREEN, I.R.; ROES-HILL, M.L.; BURTON, S.G. Enzymatic modification of 2,6-dimethoxyphenol for the synthesis of dimers with high antioxidant capacity. Process Biochemistry, v. 47, p. 1926-1932, 2012 b. 
AGRAWAL, K.; CHATURVEDI, V.; VERMA, P. Fungal laccase discovered but yet undiscovered. Bioresources and Bioprocessing, v. 5(4), p. 1-12, 2018.

ALVES, C.Q.; DAVID, J.M.; DAVID, J.P.; BAHIA, M.V.; AGUIAR, R.M. Métodos para determinação de atividade antioxidante in vitro em substrato orgânicos. Química Nova, v.33 (10), p. 2202-2210, 2010.

AMIN, R.; KHORSHIDI, A.; SHOJAEI, A.F.; REZAEI, S.; FARAMARZI, M.A. Immobilization of laccase on modified $\mathrm{Fe}_{3} \mathrm{O}_{4} @ \mathrm{SiO}_{2} @$ Kit-6 magnetite nanoparticles for enhanced delignification of olive pomace bio-waste. International Journal of Biological Macromolecules, v. 114, p. 106-113, 2018.

ASGHER, M;, WAHAB, A.; BILAL, M.; IQBAL, H.M.N. Delignification of lignocellulose biomasses by alginate-chitosan immobilized laccase produced from Trametes versicolor IBL04. Waste Biomass Valor, v. 9, p. 2071-2079, 2018.

BARRIOS-ESTRADA, C., ROSTRO-ALANIS, M. DE J., MUÑOZ-GUTTIÉRREZ, B.D., IQBAL, H.M.N., KANNAN, S., PARRA-SALDÍVAR, R. Emergent contaminants: endocrine disruptors and their laccase-assisted degradation - a review. Science of The Total Environment, v. 612, p. 1516-1531, 2018.

BENZIE, I.F.F.; STRAIN, J.J. The ferric reducing ability of plasma (FRAP) as a measure of antioxidant power: the frap assay. Analytical Biochemistry, v. 239, p. 70-76, 1996.

BILAL, M.; RASHEEDB, T.; NABEELB, F.; IQBALC, H.M.N.; ZHAO, Y. Hazardous contaminants in the environment and their laccase-assisted degradation - A review. Journal of Environmental Management, v. 234, p. 253-264, 2019.

BOUABIDI, Z.B.; EL-NAAS, M.H.; ZHANG, Z. Immobilization of microbial cells for the biotreatment of wastewater: A review. Environmental Chemistry Letters, v. 17, p. 241-257, 2019.

BRUGNARI, T.; PEREIRA, M.G.; BUBNA, G.A.; FREITAS, E.N.; CONTATO, A.G.; CORRÊA, R.C.G.; CASTOLDI, R.; SOUZA, C.G.M.; POLIZELI, M.L.T.M.; BRACHT, A.; PERALTA, R.M. A highly reusable MANAE-agarose-immobilized Pleurotus ostreatus laccase for degradation of bisphenol A. Science of the Total Environment, v. 634, p. 1346-1351, 2018.

CABALlERO, S.J.; GUERRERO, M.A.; VARGAS, L.Y.; ORTIZ, C.C.; CASTILLO, J.J.; GUTIÉRREZ, J.A.; BLANCO, S. Electroanalytical determination of catechol by a biosensor based on laccase from Aspergillus oryzae immobilized on gold screen-printed electrodes. Journal of Physics: Conference Series, v. 1119(1), 012009d, 2018.

CHAUHAN, P.S.; BINDI GORADIA, B.; SAXENA, A. Bacterial laccase: recent update on production, properties and industrial applications. 3 Biotech, v. 7, p. 323-343, 2017.

DAGLIA, M. Polyphenols as antimicrobial agents. Current Opinion in Biotechnology. V. 23, p. $174-181,2012$ 
DARONCH, N.A.; KELBERT, M.; PEREIRA, C.S.; ARAÚJO, P.H.H.; OLIVEIRA, D. Elucidating the choice for a precise matrix for laccase immobilization: A review. Chemical Engineering Journal, v. 397, 125506, 2020.

DEGÁSPARI, C.H.; WASZCZYNSKYJ, N. Propriedades antioxidantes de compostos fenólicos. Visão Acadêmica, v. 5(1), p. 33-40, 2004.

DEVASIA, S.; NAIR, J.A. Screening of potent laccase producing organisms based on the oxidation pattern of different phenolic substrates. International Journal of Current Microbiology and Applied Sciences, v. 5, p. 127-137, 2016.

DHIMAN, K.; SHIRKOT, P. Bioprospecting and molecular characterization of laccase producing bacteria from paper mills of Himachal Pradesh. Proceedings of the National Academy of Sciences, India Section B: Biological Sciences, v. 85, p. 1095-1103, 2015.

GAVNHOLT, B.; LARSEN, K. Molecular biology of plant laccases in relation to lignin formation. Physiologia Plantarum, v.116. p. 273-280, 2002.

HEIM, K.E.; TAGLIAFERRO, A.R.; BOBILYA, D.J. Flavonoid antioxidants: Chemistry, metabolism and structure-activity relationships. Journal of Nutritional Biochemistry, v. 13, p. 572-584, 2002.

HELENO, S.A.; MARTINS, A.; QUEIROZ, M.P.R.P.; FERREIRA, I.C.F.R. Bioactivity of phenolic acids: metabolites versus parent compound: A review. Food Chemistry, v. 173, p. 501-513, 2015.

HOU, J-J.; YANG, X-Q.; FU, S-R.; WANG, M-P.; XIAO, F. Preparation of double-network tofu with mechanical and sensory toughness. International Journal of Food Science Technology, v. 51, p. 962-969, 2016.

JANUSZ, G.; PAWLIK, A.; SWIDERSKA-BUREK, U.; POLAK, J.; SULEJ, J.; JAROSZWILKOŁAZKA, A.; PASZCZYNSKI, A. Laccase properties, physiological functions, and evolution. International Journal of Molecular Sciences, v. 21(966), p. 1-25, 2020.

JEON, J-R.; KIM, E-J.; MURUGESAN, K.; PARK, H-K.; KIM, Y-M.; KWON, J-H.; KIM, W-G.; LEE, J-Y.; CHANG, Y-S. Laccase-catalysed polymeric dye synthesis from plantderived phenols for potential application in hair dyeing: Enzymatic colourations driven by homo- or hetero-polymer synthesis. Microbial Biotechnology, v. 3, p. 324-335, 2010.

KATO, C.G.; GONÇALVES, G.A.; PERALTA, R.A.; SEIXAS, F.A.V.; DE SÁNAKANISHI, A.B.; BRACHT, L. COMAR, J.F.; BRACHT, A.; PERALTA, R.M. Inhibition of-amylases by condensed and hydrolysable tannins: focus on kinetics and hypoglycemis actions. Enzyme Research, v. 2017, p. 1-17, 2017.

KURISAWA, M.; CHUNG, J.E.; UYAMA, H.; KOBAYASHI, S. Enzymatic synthesis and antioxidant properties of poly(rutin). Biomacromolecules, v. 4, p. 1394-1399, 2003.

LETTERA, V.; PEZZELLA, C.; CICATIELLO, P.; PISCITELli, A.; GIACOBELli, V. G.; GALANO, E.; AMORESANO, A.; SANNIA, G. Efficient immobilization of a fungal laccase and its exploitation in fruit juice clarification. Food Chemistry, v. 196, p. 1272-1278, 2016. 
LIU, Y.Y.; ZENG, Z.T.; ZENG, G.M.; TANG, L.; PANG, Y.; LI, Z.; LIU, C.; LEI, X.X.; WU, M.S.; REN, P.Y.; LIU, Z.F.; CHEN, M.; XIE, G.X. Immobilization of laccase on magnetic bimodal mesoporous carbon and the application in the removal of phenolic compounds. Bioresource Technology, v. 115, p. 21-26, 2012.

MAMEDE, M.E.O.; PASTORE, G.M. Compostos fenólicos do vinho: estrutura e ação antioxidante. Boletim CEPPA, v. 22(2), p. 233-252, 2004.

MOGHARABI, M.; FARAMARZI, M.A. Laccase and Laccase-Mediated Systems in the Synthesis of Organic Compounds. Advanced Synthesis \& Catalysis, v. 356, p. 897-927, 2014.

MOHTAR, L.G.; ARANDA, P.; MESSINA, G.A.; NAZARENO, M.A.; PEREIRA, S.V.; JULIO RABA, J.; BERTOLINO, F.A. Amperometric biosensor based on laccase immobilized onto a nanostructured screen-printed electrode for determination of polyphenols in propolis. Microchemical Journal, v. 144, p. 13-18, 2019.

MUNIZ-MOURO, A.; OLIVEIRA, I.M.; GULLÓN, B.; LÚ-CHAU, T.A.; MOREIRA, M.T.; LEMA, J.M.; EIBES, G. Comprehensive investigation of the enzymatic oligomerization of esculin by laccase in ethanol : water mixtures. RSC Advances, v. 7, p. 38424-34833, 2017.

MUÑIZ-MOURO, A.; GULLÓN, B.; LÚ-CHAU, T.; MOREIRA, M.; LEMA, J.; EIBES, G. Laccase Activity as an Essential Factor in the Oligomerization of Rutin. Catalysis, v. 8(321), p. 1-15, 2018.

NAGHDI, M.; TAHERAN, M.; BRAR, S.K.; KERMANSHAHI-POUR, A.; VERMA, M.; SURAMPALLI, R.Y. Fabrication of nanobiocatalyst using encapsulated laccase onto chitosannanobiochar composite. International Journal of Biological Macromolecules, v. 1, p. 530-536, 2019.

NARAYANAN, M.P.; MURUGAN, S.; EVA, A.S.; DEVINA, S.U.; KALIDASS, S. Application of immobilized laccase from Bacillus subtilis MTCC 2414 on decolourization of synthetic dyes. Research Journal of Microbiology, v. 10, p. 421-432, 2015.

NEMADZIVA, B.; ROES-H, M. L.; KOORBANALLY, N.; KUDANGA, T. Small laccasecatalyzed synthesis of a caffeic acid dimer with high antioxidant capacity. Process Biochemistry, v. 69, p. 99-105, 2018.

PEZZELlA, C.; GUARINO, L.; PISCITELli, A. How to enjoy laccases. Cellular and Molecular Life Sciences, v. 72, p. 923-940, 2015.

POLAK, J.; JAROSZ-WILKOLAZKA, A. Fungal laccases as green catalysts for dye synthesis. Process Biochemistry, v. 47, p. 1295-1307, 2012.

PREHN, R.; GONZALO-RUIZ, J.; CORTINA-PUIG, M. Electrochemical detection of polyphenolic compounds in foods and beverages. Current Analytical Chemistry, v. 8(4), p. 472-484, 2012.

RATANAPONGLEKA, K.; PUNBUT, S. Removal of acetaminophen in water by laccase immobilized in barium alginate. Environmental Technology, v. 39(3), p. 336-345, 2017. 
REANO, A. F.; PION, F.; DOMENEK, S.; DUCROT, P-R.; ALLAIS, F. Chemo-enzymatic preparation and characterization of renewable oligomers with bisguaiacol moieties: promising sustainable antiradical/antioxidant additives. Green Chemistry, v. 18(11), p. 3334-3345, 2016.

REZAEI, S.; SHAHVERDI, A.R.; FARAMARZI, M.A. Isolation, one-step affinity purification, and characterization of a polyextremotolerant laccase from the halophilic bacterium Aquisalibacillus elongatus and its application in the delignification of sugar beet pulp. Bioresource Technology, v. 230, p. 67-75, 2017.

RIVERA-HOYOS， C.M.; MORALES-ÁLVAREZ， E.D.; POUTOU-PIÑALES， R.A.; PEDROSA-RODRIGUEZ, A.M.; RODRIGUEZ-VÁSQUEZ, R.; DELGADO-BOCADA, J.M. Fungal laccases. Fungal Biology Reviews, v. 27, p. 67-82, 2013.

SHARMA, A.; JAIN, K.K.; JAIN, A.; KIDWAI, M.; KUHAD, R.C. Bifunctional in vivo role of laccase exploited in multiple biotechnological applications. Applied Microbiology and Biotechnology, v. 102, p. 10327-10343, 2018.

SINGH, B.; SINGH, J; KAUR, A.; SINGH, N. Phenolic compounds as beneficial phytochemicals in pomegranate (Punica granatum L.) peel: A review. Food Chemistry, v. 261, p. 75-86, 2018.

SOARES, S.E. Ácidos fenólicos como antioxidantes. Revista de Nutrição, v. 15(1), p. 71-81, 2002.

SUCUPIRA, N.R.; SILVA, A.B.; PEREIRA, G.; COSTA, J.N. Métodos para determinação da atividade antioxidante de frutos. Ciências Biológicas e da Saúde, v. 14 (4), p. 263-269, 2012.

TAHERAN, M.; NAGHDI, M.; BRAR, S.K.; KNYSTAUTAS, E.J.; VERMA, M.; SURAMPALLI, R.Y. Degradation of chlortetracycline using immobilized laccase on polyacrylonitrile-biochar composite nanofibrous membrane. Science of The Total Environment, v. 605-606, p. 315-321, 2017.

VIDAL-LIMON, A.; SUAŔEZ, P.C.G.; ARELLANO-GARCÍA, A.; CONTRERAS, O.E.; AGUILA, S.A. Enhanced Degradation of Pesticide Dichlorophen by Laccase Immobilized on Nanoporous Materials: A Cytotoxic and Molecular Simulation Investigation. Bioconjugate Chemistry, v. 29, p. 1073-1080, 2018.

VELASCO-LOZANO, S.; LÓPEZ-GALLEGO, F.; MATEOS-DÍAZ, J.C.; FAVELATORRES, E. Cross-linked enzyme aggregates (CLEA) in enzyme improvement - A review. Biocatalysis, v. 1, p. 166-177, 2015.

XIAO, J. Dietary flvonoid aglycones and ther glycosides: Which show better biological significance? Critical Reviews in Food Science and Nutrition, v. 57(9) , p. 1874- 1905, 2017.

XIAO, J.; CAPANOGLU, E.; JASSBI, A.R.; MIRON, A. Advance on the flavonoid Cglycosides and health benefits. Critical Reviews in Food Science and Nutrition, v. 56, p. 2945, 2016. 
WANG, J.; FENG, J.; JIA, W.; CHANG, S.; LI, S.; LI, Y. Lignin engineering through laccase modification: A promising field for energy plant improvement. Biotechnol. Biofuels, v. 8(145), p. 1-11, 2015.

WELLINGTON, K.W.; GOVINDJEE, V.P.; STEENKAMP, P. A laccase- catalysed synthesis of triaminated cyclohexa-2,4-dienones from catechol. Journal of Catalysis, v. 368, p. 306$314,2018$.

ZHANG, J.; CHEN, H.; CHEN, M.; REN, A.; HUANG, J.; WANG, H.; ZHAO, M.; FENG, Z. Cloning and functional analysis of a laccase gene during fruiting body formation in Hypsizygus marmoreus. Microbiological Research, v. 179, p. 54-63, 2015.

ZHOU, Z.; HARTMANN, M. Progress in enzyme immobilization in ordered mesoporous materials and related applications. Chemical Society Reviews, v. 42(9), p. 3894-3912, 2013.

ZIYATDINOVA, G.; SNEGUREVA, Y.; BUDNIKOV, H. Novel approach for the voltammetric evaluation of antioxidant activity using DPPH - modified electrode. Electrochimica Acta, v. 247, p. 97-106, 2017. 